\title{
Is there an amodal "semantic" area in the left posterior temporal
}

\section{lobe?}

\author{
Feng Rong ${ }^{1}$, Jiang $\mathrm{Xu}^{2}$, Karen Emmorey ${ }^{3}$, Allen R. Braun ${ }^{2}$, Gregory Hickok ${ }^{1}$
}

1. Auditory \& Language Neuroscience Lab, Center for Cognitive Neuroscience, Department of Cognitive Sciences, University of California, Irvine. Irvine, CA 92697.

2. Language Section, National Institute on Deafness and Other Communication Disorders, National Institutes of Health. Bethesda, MD 20892.

3. Laboratory for Language and Cognitive Neuroscience, San Diego State University. San Diego, CA 92120

\begin{abstract}
Author note: This manuscript was submitted for publication in Psychonomic Bulletin \& Review and rejected in Jan 2015. Reviews are appended to this manuscript. The principle reason, which we agree with, is that we did not frame the work sufficiently well in the context of current theories of semantic organization of the brain. Our intent, however, was simply to clarify a previously published result and not delve into all the theoretical details of semantic systems in cortex. The methodological concerns raised by one reviewer are easily addressed; see our responses in the reviewer appendix. In the end, the project was not of sufficient priority to warrant the work necessary to get it published. Now with the advent of manuscript repositories we have decided to make the work public.
\end{abstract}




\begin{abstract}
Portions of the left posterior temporal lobe are widely believed to comprise a “convergence zone" for integrating information from various sensory modalities and therefore for accessing high-level semantic information. It is an open question whether the network in this posterior temporal lobe region is coding/processing abstract, amodal information or whether neural codes retain sensory modality-tied representations. A recent functional MRI study (Xu et al. 2009, PNAS, 106, 20664-20669) seems to support the amodal account: using standard univariate general linear model (GLM) analyses, the left posterior temporal lobe region was equally activated during the processing of visually presented meaningful symbolic hand gestures and auditorily presented spoken glosses of those same gestures, relative to meaningless control gestures/speech, thus showing no preferential response according to modality of presentation of the same information. However, standard univariate analyses may not be sensitive to the microorganization of the region of interest. In the present study we reanalyzed the same dataset using a region of interest (ROI)-based multivariate pattern classification method to assess modality sensitivity in the posterior temporal lobe ROI. We found that the pattern of activity in the ROI significantly discriminated between the visual and auditory conditions, including the meaningless control conditions. Moreover, mapping of the prediction coefficients onto the voxels within the ROI demonstrated a gradient of preference among modalities, where the gesture preferring voxels were distributed more medial and posterior, and the speech preferring voxels were distributed more lateral and anterior. These findings suggest that the left posterior temporal region is not coding information in an amodal format, at least broadly, but contains sub-regions that are sensitive to each individual modality.
\end{abstract}




\section{Introduction}

Portions of the left posterior temporal lobe (e.g., cortex in the posterior middle temporal gyrus, pMTG) are widely viewed as a "convergence zone" or as a kind of integration hub for lexical semantic processing (Binder et al., 1997; Damasio, 1989a; Dronkers, Wilkins, Valin, Redfern, \& Jaeger, 2004; Hickok \& Poeppel, 2007; Rissman, Eliassen, \& Blumstein, 2003; Rodd, Davis, \& Johnsrude, 2005). For example, a recent meta-analysis by Binder and colleagues (Binder, Desai, Graves, \& Conant, 2009) identified cortex in the lateral ventral temporal lobe including the MTG as part of the brain's semantic network, and conclude that, "these regions [...] are likely heteromodal cortex involved in supramodal integration and concept retrieval" (p. 2776). Binder et al. (2009) also note, as do Hickok \& Poeppel (2007), that damage to the posterior MTG has been associated with language comprehension and semantic deficits (Chertkow, Bub, Deaudon, \& Whitehead, 1997; Dronkers et al. 2004; Hart \& Gordon, 1990; Hillis \& Caramazza, 1991; Kertesz, Lau, \& Polk, 1993).

This same region has been found to activate similarly in fMRI studies during the comprehension of meaningful information in both the auditory and visual modalities (Dick, Mok, Beharelle, Goldin-Meadow, \& Small, 2014; Straube, Green, Bromberger, \& Kircher, 2011; Xu, Gannon, Emmorey, Smith, \& Braun, 2009). One study in particular directly compared auditory spoken language stimuli with visual gestural stimuli (pantomimes and emblems) that communicated the same semantic information. The left posterior MTG (as well as an inferior frontal region) was activated in both conditions and in fact responded to the auditory and visual stimuli to a degree that was statistically indistinguishable using a univariate general linear model (GLM) (Xu et al., 2009). From this finding, it was concluded that, “ inferior frontal and posterior temporal areas correspond to an amodal system [...] that plays a central role in human 
communication — a semiotic network in which meaning (the signified) is paired with symbols (the signs) whether these are words, gestures, images, sounds, or objects" (p. 20668).

Such findings as those reported in Binder et al.'s meta-analysis of high-level semantic processing as well as Xu et al.'s more focused study of communicative speech versus manual gestures are consistent with the view that cortex in posterior MTG region is processing and/or representing information in an amodal fashion, that is, that the same neural ensembles code abstract semantic information independently of modality (Damasio, 1989b; Rogers \& McClelland, 2004). But it is also possible that the region is better characterized as "heteromodel", that rather than coding abstract, amodal representations, the posterior MTG contains intermixed modality-dependent neural ensembles that support cross-modal integration of more widely distributed modality-specific semantic information (Binder et al. 2009; Binder \& Desai, 2011; Hickok \& Poeppel, 2007). For example, fMRI responses that appear to be amodal in that they fail to distinguish between information coming from different sensory modalities may be a consequence of insufficient measurement sensitivity rather than a direct reflection of the organization of the system.

The present study sought to explore this issue using multivariate pattern analysis (MVPA) rather than standard GLM analysis of the fMRI data. MVPA relies on the fact that due to the spatial resolution of fMRI, each voxel contains thousands of neurons, each potentially tuned to different features of the stimulus or process, and that the distribution of "tuning functions" of the populations of neurons across voxels is not uniform within a region. Thus, by looking at the pattern of activation across voxels within a region instead of contrasting the response amplitude for each single voxel, it is possible to detect underlying differences in the response to different stimulus conditions using MVPA, even in cases where the univariate linear 
analysis might result in non-significant contrast between the modalities (Haxby et al. 2001; Kriegeskorte, Goebel, \& Bandettini, 2006; Norman, Polyn, Detre, \& Haxby, 2006).

In this study, we re-analyzed the dataset from Xu et al. (2009) and assessed the modality specificity in left posterior temporal lobe utilizing an ROI-based MVPA method (Okada et al. 2010). Our approach is first to replicate the original results in a new re-analysis of the data using a standard GLM and then to use the GLM findings to define ROIs in which we can carry out MVPA analyses. To preview our findings, our re-analysis using the GLM approach identified the posterior MTG site as a region that responds in a statistically indistinguishable manner to meaningful speech and gesture stimuli, but our more conservative methods failed to confirm the inferior frontal region. Turning our focus to the posterior MTG ROI we found that MVPA successfully discriminated the speech and gesture conditions and in fact contains an anteriorposterior gradient among the voxels reflecting sensitivity to the auditory and visual modalities respectively. 


\section{Materials and Methods}

\section{Data Source}

Using an ROI-based classification method, we reanalyzed the fMRI dataset of Xu et al. (2009), which consisted of BOLD signals from twenty right-handed healthy adult participants. Each participant was scanned in five sessions during which stimuli were presented in randomized 45-second blocks. The stimuli included video clips of (1) pantomime gestures (e.g., opening a jar), (2) emblem gestures (e.g., "thumbs up"), (3) nonsense gestures, English spoken equivalents (semantic glosses) of the (4) pantomime and (5) emblem stimuli, and (6) scrambled pseudowords as the equivalent of the nonsense gestures. Stimuli were presented as $1.5 \mathrm{~s}$ video clips; for speech stimuli, the video showed the actress speaking the utterance. Each block contained fifteen stimuli of a given type with a $3 \mathrm{~s}$ inter-stimulus-interval (ISI).

As stated in $\mathrm{Xu}$ et al. (2009), whole brain BOLD images 'were acquired on a 3T GE scanner using a gradient echo $\mathrm{EPI}$ sequence $\left(\mathrm{TR}=2000 \mathrm{~ms}, \mathrm{TE}=30 \mathrm{~ms}, \mathrm{FA}=90^{\circ}, \mathrm{FOV}=240\right.$ x $240 \mathrm{~mm}$, matrix size $=64 \times 64$, voxel size $\left.=3.75 \times 3.75 \times 6 \mathrm{~mm}^{3}\right)$.' Each volume contained twenty-four axial slices without gap.

\section{Data Analysis}

ROI Identification. The dataset from Xu et al. (2009) was reanalyzed to take advantage of new statistical methods for functional image analysis developed since the report's publication. We first identified the brain regions that were active during processing meaningful gesture symbols and corresponding spoken glosses. Using AFNI software (Cox, 1996), each subject's motion-corrected and spatially aligned BOLD signals were normalized to a standard EPI template in Talairach space and resampled into $3 \mathrm{~mm}$ isotropic voxels. The transformed signals were then smoothed with a Gaussian kernel of $9 \mathrm{~mm}$ full width at half maximum, high-pass 
filtered with stop frequency at $0.008 \mathrm{~Hz}$, and scaled relative to the mean. After preprocessing, we applied regression analysis on the datasets for each subject with 6 task related regressors (pantomime gestures - GP, emblem gestures -- GE, nonsense control gestures -- GC, spoken pantomime glosses - SP, spoken emblem glosses -- SE, and scrambled spoken pseudowords -SC) and 6 motion regressors. The resulting coefficients and t-statistics for each experimental condition were then input into the group level analysis using a mixed-effect meta-analysis method (Chen et al., 2012). We then identified the clusters of voxels that showed greater response to meaningful stimuli in either modality compared to their non-meaningful controls. Specifically, we identified voxels that responded to the two meaningful gesture conditions $(\mathrm{GP}+\mathrm{GE})$ compared to the nonsense gesture controls $(\mathrm{GC})$ and to the two meaningful speech conditions ( $\mathrm{SP}+\mathrm{SE})$ compared to the scrambled speech condition (SC). The statistical threshold was set at $\mathrm{p}<0.05$ after being corrected for family-wise errors (FWE; with uncorrected $\mathrm{p}<0.005$, and corresponding threshold of cluster size that has been obtained using a Monte Carlo method, which calculates the threshold cluster size by taking into account the variance of each value set among the voxels; Forman et al. 1995). This process was similar to the conjunction analysis procedure employed by Xu et al. (2009) to identify regions that responded to meaningful gestures and speech compared to controls, with the following exceptions: (1) As we have mentioned above, for group level analysis, we used a recently developed mixed-effect metaanalysis (MEMA) method, which takes into account the individual-level variability of the effect of interest and eases the strong assumption that the within-subject variances among individuals are the same. In brief, MEMA weights each subject's contribution to the group level analysis according to the reliability of that subject's effect estimate (i.e., confidence interval). (2) We applied a more conservative approach on the statistical evaluation of significance for the 
conjunction analysis - only clusters showing statistical significance in both contrasts were included for following ROI-based MVPA analysis. This is an approach commonly used in analysis using AFNI, and is different from the approach in SPM (Friston, Penny, \& Glaser, 2005), which was used for data analysis in Xu et al. (2009).

ROI-based Multivariate Pattern Analysis (MVPA). After identification of the ROI, we used an ROI-based multivariate pattern analysis (MVPA) method (Okada et al. 2010) that employed a linear support vector machine (SVM) algorithm (Vapnik, 1995) to test whether the voxels within the identified ROI(s) respond differently to the various conditions. The analysis tools were custom-developed using MATLAB (The MathWorks, Inc., Natick, MA) and an open source library for SVM (LIBSVM; Chang \& Lin, 2011). Within the identified ROI, we performed five pair-wise SVM classification analyses: (1) all gesture conditions versus all speech conditions including controls (GP+GE+GC vs. SP+SE+SC); (2) meaningful symbolic gestures versus meaningful spoken glosses (GP+GE vs. SP+SE); (3) non-meaningful gesture control versus scrambled speech (GC vs. SC); (4) pantomime versus emblem gestures (GP vs. GE) and (5) spoken glosses describing pantomime versus emblem gestures (SP vs. SE). Note that these analyses are orthogonal to the ROI definition procedure. Any pattern analysis that showed significant classification results suggests that the activity pattern among voxels within this ROI could distinguish the two compared experimental conditions.

Each SVM analysis was performed on the individual subject level using spatially unsmoothed data. Before applying the SVM, we preprocessed the BOLD signal by normal scaling and averaging: First, we extracted the motion-corrected, spatially aligned, template registered, and linearly detrended BOLD signal from the voxels within the ROI identified with the group-level GLM process (see above), then normally scaled the signal from different scans 
into the same data range by calculating z-scores for each voxel. Second, we averaged the normally scaled signal across the volumes in each of the two experimental trials to obtain a \#trial by \#voxel matrix for each scanning session. To compensate for the discrepancy between TR (2s) and length of each trial (3s), we averaged signal from two consecutive trials to get a 'pseudo-trial average' for further MVPA analysis. We then performed the linear SVM analyses on the preprocessed dataset with a leave-one-out cross validation approach (Vapnik, 1995). For each of the leave-one-out iterations, we trained the classifier with preprocessed signal from the experimental trials of the compared conditions across all scanning sessions except one session. We next used the trained classifier to test the left out session and calculated a d' value based on the classification results to evaluate the performance as follows:

$$
d^{\prime}=Z(P(H i t))-Z(P(F A))
$$

$\mathrm{P}(\mathrm{Hit})$ is the probability of 'hit' trials, which equals the proportion of trials that were correctly classified as one of the conditions (e.g., proportion of trials that were classified as gestures and were indeed gestures), and $\mathrm{P}(\mathrm{FA})$ is the probability of 'false alarm' trials, which equals to the proportion of trials that were incorrectly classified as the same condition (e.g., proportion of trials that were classified as gestures but were actually spoken glosses). $Z(p)$ returns the ztransform of the corresponding probability values.

For each comparison, we quantified the classification performance by averaging the d' values across the five leave-one-out sessions, and then statistically evaluated the significance of these classification results using a nonparametric bootstrap method (Lunneborg, 2000): similar SVM procedures were repeated 10,000 times for each pair-wise classification by randomly reshuffling the condition labels in the training dataset and testing the trained classifier using the remaining test dataset with real condition labels in each leave-one-out iteration. Therefore, we 
obtained a bootstrapped distribution of the 'null' d' values, in which the values are normally distributed with the mean at the chance level of zero. To assess classification performance for each participant in each contrast, we performed a within-subject nonparametric statistical analysis by testing against the null hypothesis whether the classification d' value equaled the mean of the bootstrap distribution by computing a one-tailed accumulated percentile of the original classification d' value in the distribution of the bootstrapped d' values. If the accumulated $\mathrm{P}>0.95$, we rejected the null hypothesis and concluded that for this participant, the trained classifier from the corresponding ROI significantly discriminated between the two tested experimental conditions. In addition to evaluating the classification performance within each individual participant, we also assessed the significance of the classification performance on the group level. For each contrast, a bootstrap-T approach is implemented using the mean d' values across all participants: in the first step we constructed a bootstrap-T distribution based upon the 'null' d' values obtained from the bootstrap iteration among all participants by performing a ttest against the group chance d' value (the mean bootstrap d' value across all participants). In a second step, we calculated the averaged d' value among all participants based on the classification results from the original data, and performed a t-test against the group chance d' value. We then computed the accumulated percentile of the t-score obtained from the second step on the bootstrap-T distribution determined by the first step. If the accumulated $\mathrm{P}>0.95$, we rejected the null hypotheses and concluded that the trained classifier from the corresponding ROI significantly discriminated between the two tested experimental conditions at the group level.

We also investigated the spatial alignment of the voxels within the identified ROI to examine whether there exists a spatial gradient among the voxels for the sensitivity of the two sensory modalities. For this purpose, we calculated the averaged predictive coefficient (w) 
values for the voxels within the identified ROI and overlaid them onto the template Talairach anatomical images. For each trained classifier, the inner product of the predictive coefficients and the averaged signal predicts the condition label of each trial during the testing phase of SVM. The logic behind this procedure is that for pair-wise linear SVM procedure, if a voxel is unilaterally sensitive to one condition, the values of its predictive coefficients will be mainly positive or negative, therefore the averaged predictive coefficient of this voxel will not be close to zero; whereas for the voxels that are jointly sensitive or insensitive to the compared conditions, the predictive coefficients should be distributed evenly on both sides among the trials, hence the averaged values should be close to zero. Therefore, if most voxels in an ROI displayed mean predictive coefficients close to zero, it is either insensitive to the compared conditions, or sensitive to both conditions equally. On the other hand, if the voxels in an ROI displayed mean predictive coefficients with at least two subgroups, one is mainly negative and another mainly positive, we can conclude that the ROI contains two subsets of voxels such that each one is sensitive to one of the compared conditions. After grouping the voxels based upon their mean predictive coefficients, we examined their spatial alignment (spatially clustered or scattered) and sensitivity to each type of stimuli. For the latter approach, we separated the ROI into three subsets of voxels based on the mean and standard deviation (std) of their predictive coefficients (NegVal: $\mathrm{w} \leq$ mean $-1 / 3$ std; InnerCore: mean $-1 / 3$ std $<\mathrm{w}<$ mean $+1 / 3$ std; and PosVal: $w \geq$ mean $+1 / 3$ std), then repeated the five above-mentioned pair-wise classification analyses on each group to assess their functional roles correlated with the spatial arrangement. 


\section{Results}

\section{ROI Identification}

Group level conjunction analyses ([1/2(GP+GE) vs. GC] AND [1/2(SP+SE) vs. SC]) using general linear model (GLM) yielded one cluster of significant voxels. Consistent with the findings in $\mathrm{Xu}$ et al. (2009), the region of interest (ROI) localizes to the left posterior temporal lobe (Figure 1A), extending from the superior temporal sulcus (STS) to the middle temporal gyrus (MTG). The ROI has 91 voxels (Talairach coordinates of the center: [-53 -46 4]), shows greater activity during processing of meaningful gestures and spoken glosses than corresponding controls (Figure 1C). It did not show a significant difference for the general linear contrast between meaningful gestures and spoken glosses (Figure 1B). 
A)

B)
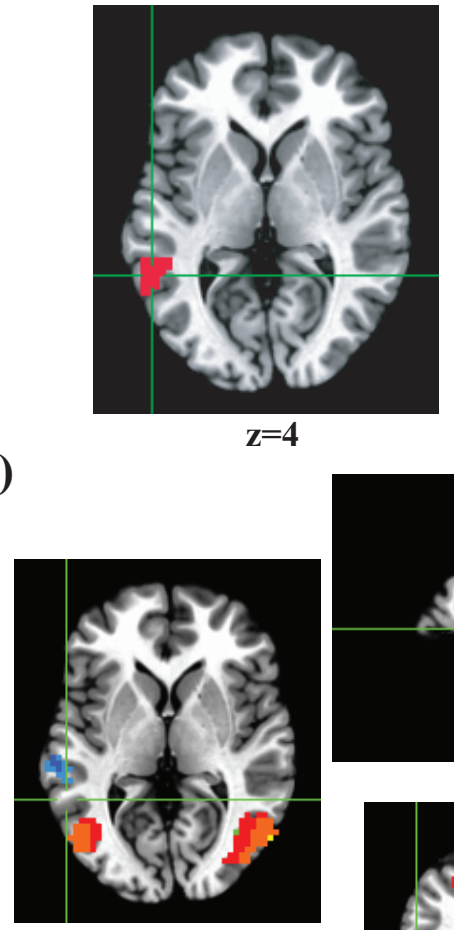

$-10$
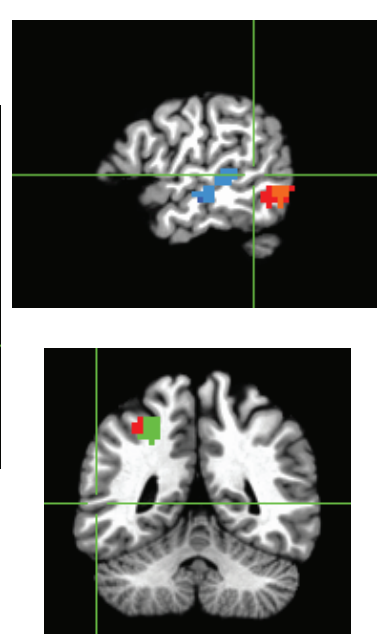

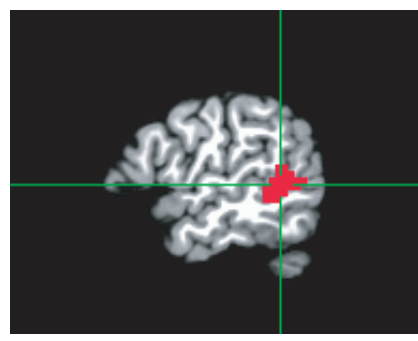

$\mathbf{x}=\mathbf{- 5 3}$

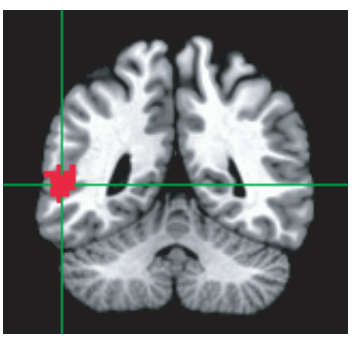

$y=-46$

C)

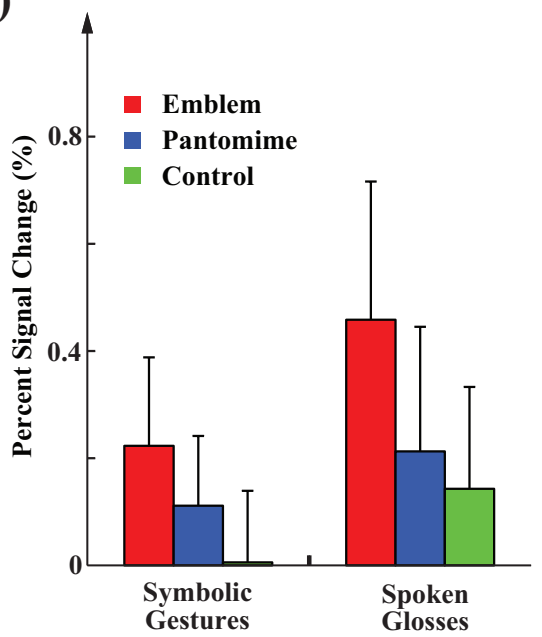

Figure 1. Group results from conjunction analysis $([1 / 2(G P+G E)$ vs. GC] AND [1/2(SP+SE) vs. SC]) based upon GLM contrasts (family-wise error corrected $p<0.05$ ). The abbreviations are: GP -- gesture pantomime; GE -- gesture emblem; GC -- gesture control; SP -- spoken pantomime; SE -- spoken emblem; SC -- spoken control. A) Axial, sagittal, and coronal slices showing the left posterior MTG/STS ROI that demonstrated significant greater response in both meaningful symbolic gesture and spoken glosses processing than corresponding controls. The axial and coronal images are in neurological convention (left =left). B) Axial, sagittal, and coronal slices showing the $t$-score map of the contrast $(G P+G E) v s$. $(S P+S E)$. Same to $A)$, the hair cross indicates the center of the left $p M T G$ cluster. $C)$ Average activities of the voxels within the ROI for all six conditions. The error bars stand for standard error of means (SEM).

\section{Classification Results}


Figure 2A presented classification results using the identified left posterior MTG/STS ROI. BOLD signals from the ROI can discriminate gestures from spoken stimuli no matter whether they were averaged across all conditions including the controls (d' $=3.04 \pm 1.494, \mathrm{p}<$ 0.001 ), averaged across pantomime and emblem without the controls (d' $=3.25 \pm 1.638, \mathrm{p}<$ $0.001)$, or just between the control stimuli $\left(\mathrm{d}^{\prime}=3.04 \pm 2.190, \mathrm{p}<0.005\right)$. In addition, bootstrap analysis on individual level SVM statistics showed that in most participants the ROI could discriminate stimuli from the two different modalities (GP+GE+GC vs. SP+SE+SC: 20/20; GP+GE vs. SP+SE: 17/20; and GC vs. SC: 13/20). While the GLM contrasts demonstrated that this region responded significantly stronger to meaningful symbolic gestures and spoken glosses than corresponding controls, it could not discriminate pantomime from emblem stimuli in either symbolic gestures $\left(d^{\prime}=0.50 \pm 1.712, p>0.05\right)$ or spoken glosses $\left(d^{\prime}=-0.63 \pm 2.288, p>0.05\right)$. 
A

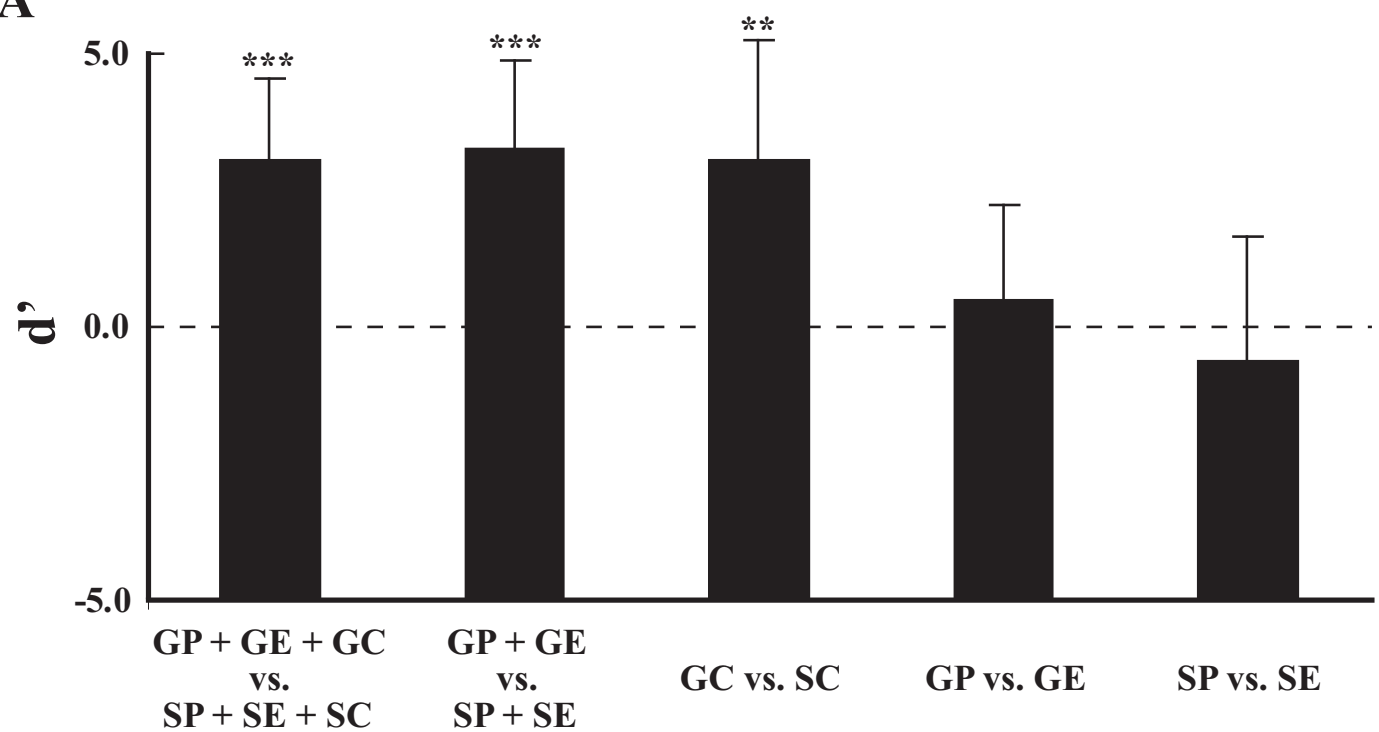

B

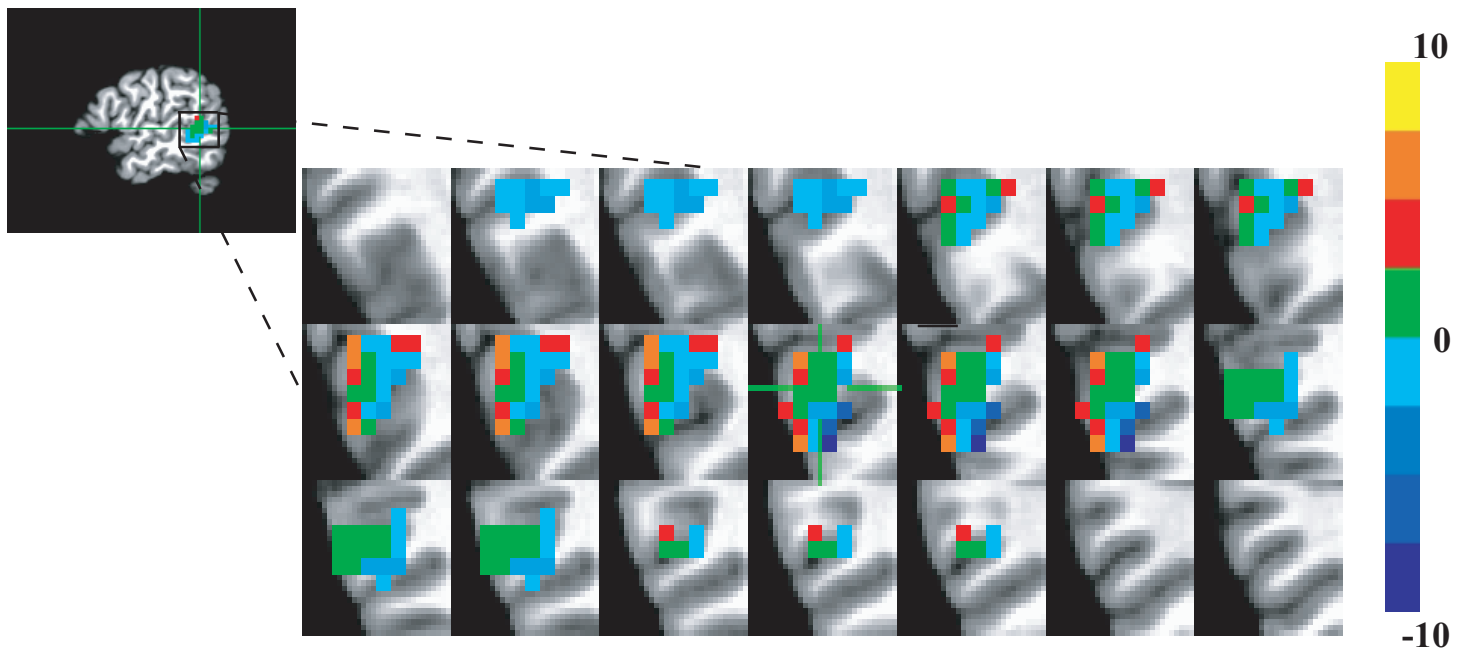

C
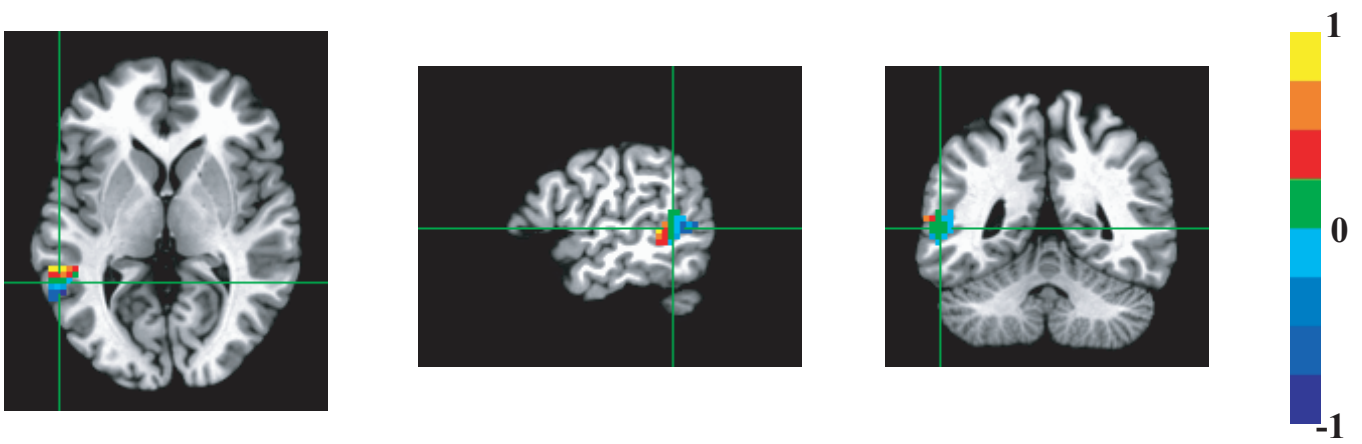

Figure 2. Pair-wise classification with the identified ROI. A) Performance of the specified pairwise classifications measured as d'values. Statistical significance was assessed by the bootstrap-T test (***: $p<0.001$; **: $p<0.005)$. The dash-line indicates the averaged chance level d'value obtained from bootstrapping. B) Axial images of the averaged predictive 
coefficient values among the voxels within the identified ROI. The values were from the trained classifier for the $(G P+G E+G C)$ vs $(S P+S E+S C)$ classification, the colder colors indicate the voxels being more selective to gestures and the warmer colors indicate the voxels being more selective to spoken glosses. The top-left inset is a sagittal slice indicating the spatial location and boundaries of the axial images. The pattern of the modality selectivity is consistent with the pattern shown in C) GLM coefficient images obtained from the same contrast. The color values stand for the group level t-scores.

Further examination of the predictive coefficient values from the trained classifiers $(\mathrm{GP}+\mathrm{GE}+\mathrm{GC}$ vs. $\mathrm{SP}+\mathrm{SE}+\mathrm{SC})$ suggested a posterior-anterior and medial-lateral gesture-speech sensitivity gradient among the voxels within the ROI. Along the gradient, the averaged predictive coefficient values changed from mostly negative to mostly positive, indicating a stimulus sensitivity gradient from gesture to spoken glosses (Figure 2B). This gradient pattern was consistent to the pattern of the coefficients obtained from the same contrast based on the GLM model (Figure 2C), although statistical analysis of the GLM contrast coefficients was not significant. To assess the functional importance of this gradient pattern and examine whether the different voxel groups -- especially the group at the center -- can classify the signals from different modalities, we separated the voxels into three groups for each participant based on the mean and standard deviations (std) of their predictive coefficients obtained from the leave-oneout iteration (NegVal: $\mathrm{w} \leq$ mean $-1 / 3$ std; PosVal: $\mathrm{w} \geq$ mean $+1 / 3$ std; and InnerCore: mean $1 / 3$ std $<\mathrm{w}<$ mean $+1 / 3$ std). With this grouping criterion, each voxel group has roughly one third of all of the voxels within the ROI (NegVal: $32 \pm 4.9$; PosVal: $33 \pm 4.5$; InnerCore: $26 \pm$ 5.2). The mean predictive coefficient values of the three groups were significantly different from each other (NegVal: $-5.9 \pm 1.81$; PosVal: $5.8 \pm 1.83$; InnerCore: $0.1 \pm 0.21$; statistically assessed by a one-way repeated measures ANOVA with Greenhouse-Geisser correction for sphericity: $\mathrm{F}(2,38)=227.3, \mathrm{p}<0.001)$. We then repeated the five above-mentioned pair-wise classifications on each voxel group to assess sensitivity to gestures and spoken stimuli. The 
classification results are presented in Table 1 and Figure 3. All voxel groups showed classification results similar to the whole ROI, even the group in the inner core, which hypothetically has the least power to discriminate gestures from spoken glosses because the mean predictive coefficients from the trained classifiers are close to zero. Nevertheless, the results indicated that neuronal activity among the voxels within this left posterior MTG/STS ROI had similar power to discriminate symbolic gestures from the spoken glosses, even though the patterns of the predictive coefficients and the GLM contrast coefficients both implied poor classification capability of the voxels locates in the middle of the ROI.

\begin{tabular}{|c|c|c|c|c|c|c|}
\hline & & $\begin{array}{c}\mathrm{GP}+\mathrm{GE}+\mathrm{GC} \\
\text { vs. } \\
\mathrm{SP}+\mathrm{SE}+\mathrm{SC}\end{array}$ & $\begin{array}{c}\mathrm{GP}+\mathrm{GE} \\
\text { vs. } \\
\mathrm{SP}+\mathrm{SE}\end{array}$ & GC vs. SC & GP vs. GE & SP vs. SE \\
\hline \multirow{5}{*}{ 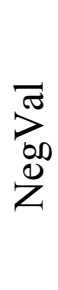 } & Mean d' & 1.97 & 1.92 & 1.76 & 0.34 & -0.54 \\
\hline & Std_d' & 0.924 & 1.091 & 2.058 & 1.178 & 1.914 \\
\hline & t-score & 9.6 & 7.92 & 3.84 & 1.31 & -1.27 \\
\hline & p-value* & $<0.001$ & $<0.001$ & $<0.05$ & $>0.05$ & $>0.05$ \\
\hline & $\begin{array}{c}\text { \# Significant } \\
\text { Subjects** }\end{array}$ & 19 & 15 & 10 & 2 & 1 \\
\hline \multirow{5}{*}{ 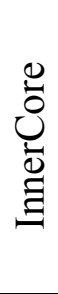 } & Mean_d' & 1.07 & 1.05 & 1.26 & 0.34 & -0.31 \\
\hline & Std_d' & 0.583 & 1.033 & 1.423 & 1.739 & 1.874 \\
\hline & t-score & 8.26 & 4.57 & 3.95 & 0.88 & -0.73 \\
\hline & $p$-value & $<0.001$ & $<0.005$ & $<0.05$ & $>0.05$ & $>0.05$ \\
\hline & $\begin{array}{c}\text { \# Significant } \\
\text { Subjects }\end{array}$ & 14 & 6 & 5 & 4 & 1 \\
\hline \multirow{5}{*}{$\sum_{0}^{\pi}$} & Mean_d' & 1.55 & 1.61 & 1.67 & 0.27 & -0.84 \\
\hline & Std_d' & 0.685 & 1.017 & 1.656 & 1.384 & 2.077 \\
\hline & t-score & 10.2 & 7.15 & 4.52 & 0.89 & -1.8 \\
\hline & $p$-value & $<0.001$ & $<0.001$ & $<0.01$ & $>0.05$ & $>0.05$ \\
\hline & $\begin{array}{l}\text { \# Significant } \\
\text { Subjects }\end{array}$ & 16 & 14 & 9 & 3 & 1 \\
\hline
\end{tabular}

Table 1. Classification results for sub-groups of voxels in the identified ROI.

* The p-value is obtained from the group-level bootstrap-T test, see method section for detail description 
A

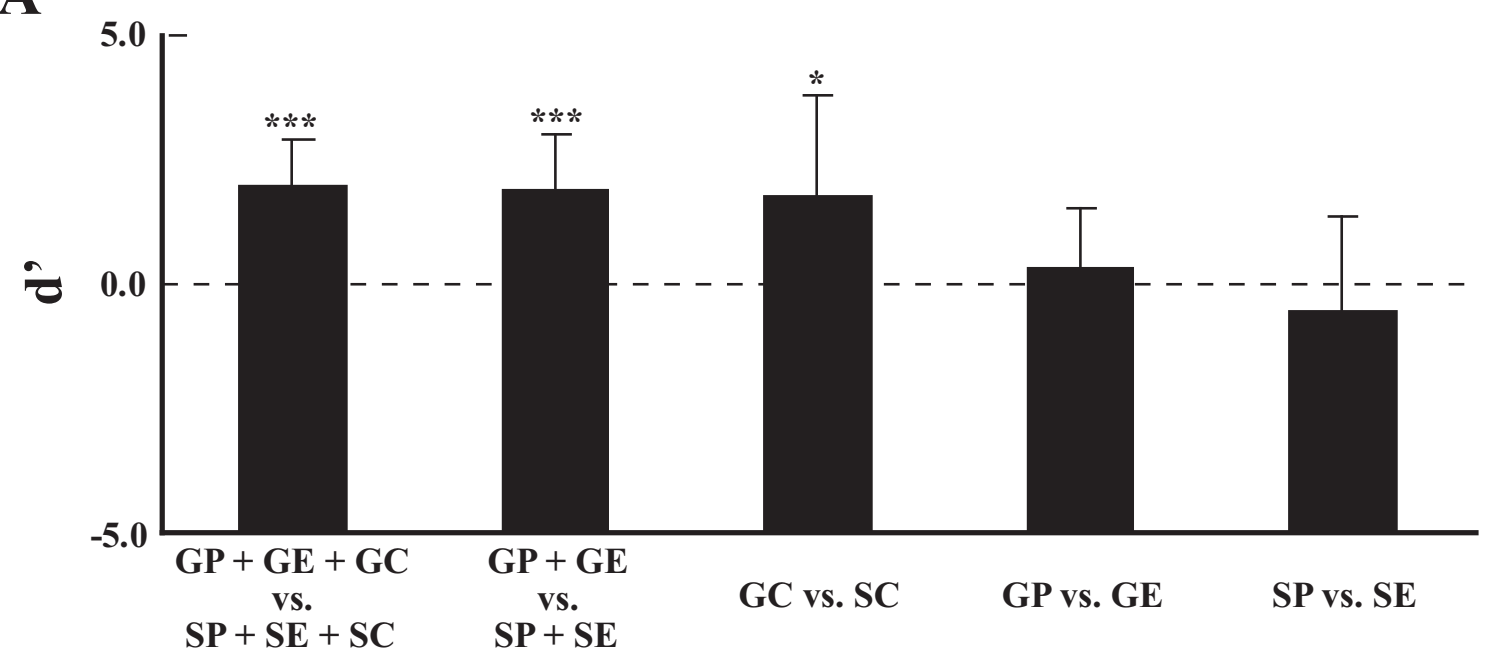

B

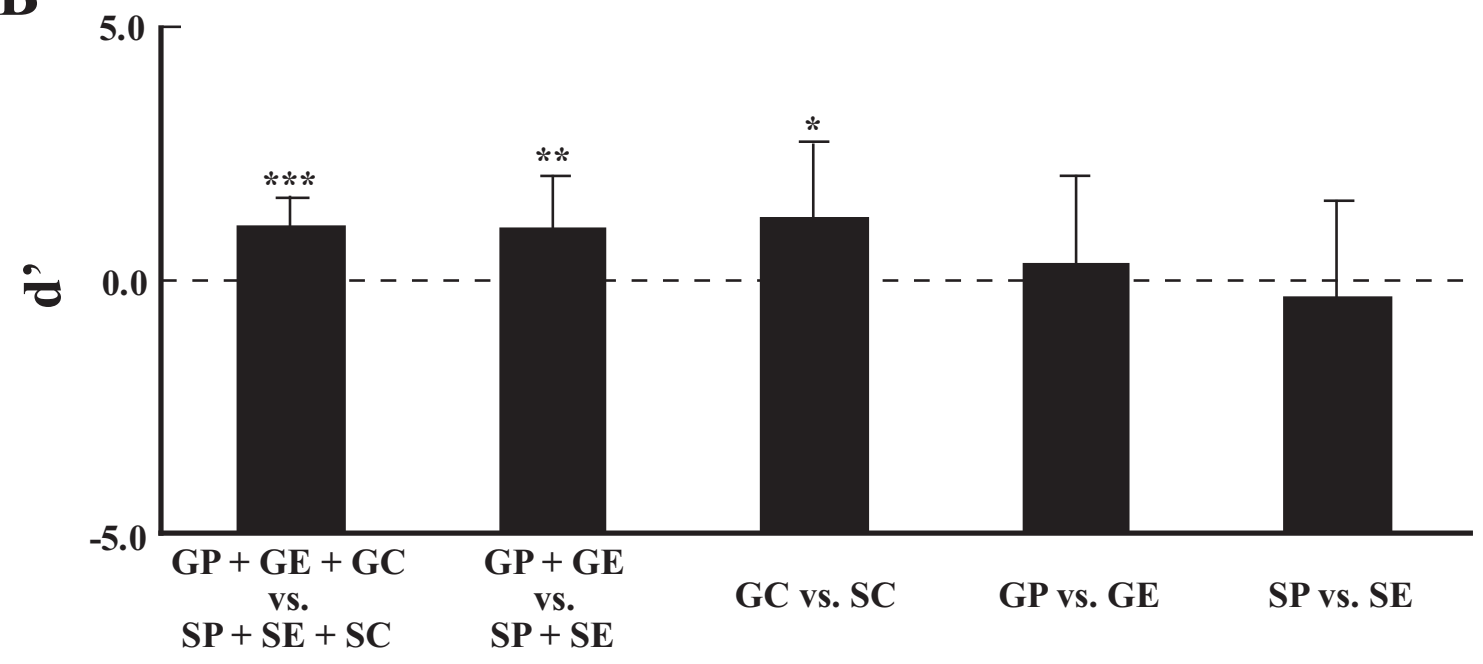

C

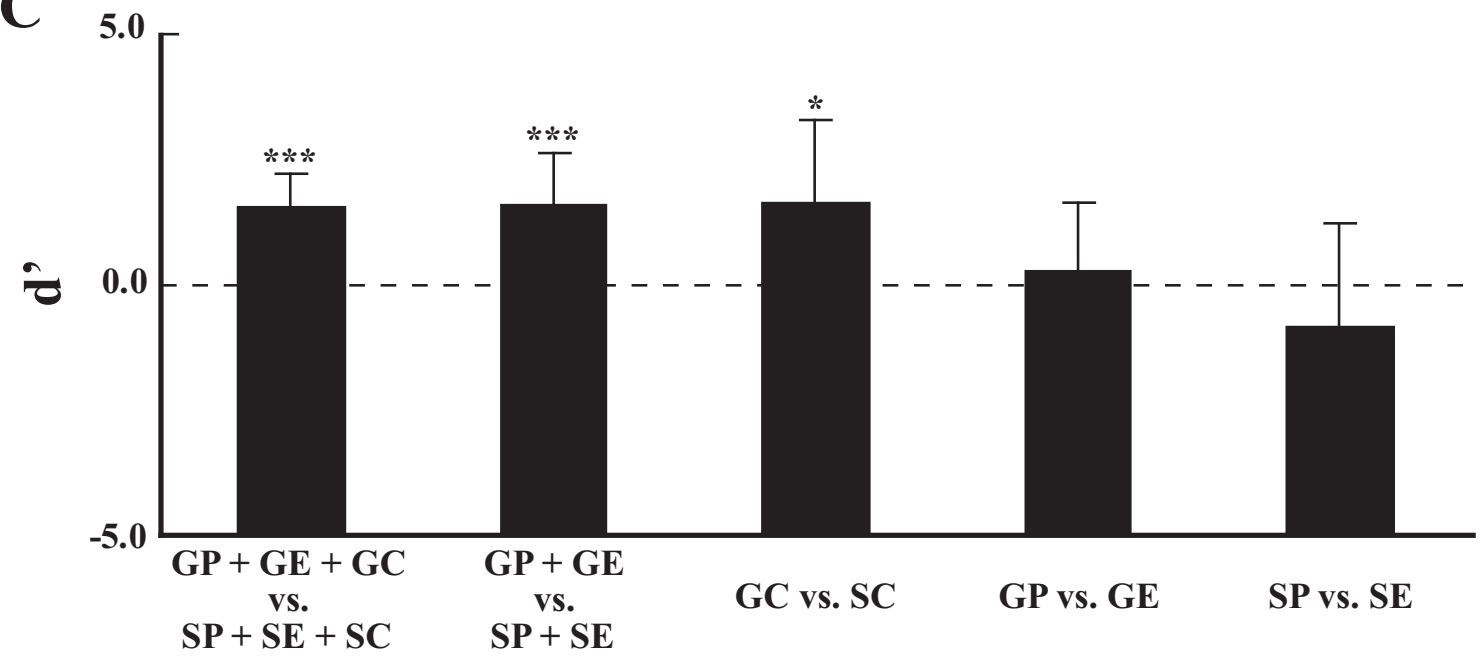


Figure 3. Performance of classifications using three separated groups of voxels within the left posterior MTG/STS ROI. A) NegVal group; B) InnerCore group; and C) PosVal group. See text for description of grouping.

\section{Discussion}

Canonical univariate analyses of BOLD signals using a general linear model demonstrated stronger responses in a left posterior temporal cluster of voxels to meaningful symbolic gestures and spoken glosses than corresponding non-meaningful control stimuli (Xu et al., 2009; as confirmed by the GLM analysis in this study). Furthermore, lack of significance in further univariate linear contrasts between responses to meaningful symbolic gestures and spoken glosses suggested that this region is insensitive to different sensory modalities during semantic processing, which is consistent with an 'amodal' functionality for comprehension of verbal and gestural communicative information. However, in this study, by applying an ROIbased multivariate pattern classification method to analyze the same dataset, we found the activity pattern among all voxels in this ROI was able to differentiate between speech and gesture signals for both meaningful and non-meaningful stimuli, whereas it could not distinguish between meaningful stimuli from two different categories (pantomime and emblem) within each modality. The modality preference among the voxels, as quantified by the mean classifier predictive coefficients, demonstrated an anterior-lateral to posterior-medial spatial gradient along the speech-preferred to gesture-preferred continuum, whereas further analyses using sub-clusters of voxels based on their modality preferences demonstrated identical discrimination capability among the sections. These results indicate that, although each single voxel's modality preference is too weak to be detected by univariate methods, patterns among all voxels within the left posterior MTG/STS are modality sensitive during processing of semantic and non-semantic information. 
One intuitive question about the current study is why GLM and MVPA analyses generate distinct results on the same dataset: with the GLM method, the left posterior temporal region responded similarly to meaningful symbolic gestures and spoken glosses and could not be distinguished from each other; whereas with the MVPA method, the spoken and gestural stimuli could be discriminated with the BOLD signals from the same set of voxels. As described in previous theoretical analyses (Haxby et al., 2001; Norman et al., 2006; Kriegeskorte et al., 2006), different models and methods are used to correlate the BOLD signal with the experimental conditions: in a GLM analysis the response waveform of each voxel is fitted with a model using the behavioral state time course as an independent variable, whereas the linear Support Vector Machine (SVM) uses the multi-voxel time course in the ROI as the independent variable, and the behavior states were fitted as the dependent variables. With these different approaches, univariate GLM analyses are sensitive to large amplitude differences between compared experimental conditions on single-voxel level. In contrast, the MVPA method can utilize both single-voxel large-amplitude differences and multiple-voxel small-amplitude pattern differences among a group of voxels to discriminate different response patterns to different experimental conditions. The latter approach is specifically sensitive to stimulation from multiple modalities in highly multimodal regions, in which each voxel might contain neurons that specifically respond to several cognitive states in ways that are hard to discriminate from each other, while the high-dimensional vector of values among all voxels corresponding to different states can be distinguished using the multivariate classifiers. Therefore, the seemingly disparate results with the mass-univariate GLM method and the multivariate pattern classification method can be reconciled with each other if we consider each voxel within the ROI as a population of thousands of neurons and some of them have biased responses to each sensory modality. Because these 
biases are relatively small, the amplitude difference of single-voxel responses to different experimental conditions cannot be detected by the mass-univariate GLM method. However, since the modality-sensitive neurons are not evenly distributed across the voxels, a vector that consists of values from a group of voxels has the potential to discriminate different experimental conditions in a much higher-dimensional space (Figure 2).

There is no doubt that the left posterior temporal region centered on the posterior middle temporal gyrus (pMTG) receives highly processed inputs from the auditory and visual modalities and participates in both linguistic and nonlinguistic semantic-level processes. For example, it has been shown to be activated during the processing of meaningful information in the form of speech (Binder, Swanson, Hammeke, \& Sabsevitz, 2008; Humphries, Binder, Medler, \& Liebenthal, 2007; Miglioretti \& Boatman, 2003;), sign language (Courtin et al., 2011; Emmorey et al., 2004), symbolic pantomime/emblem gestures (Xu et al., 2009) and coverbal iconic/metaphoric gestures (Dick et al. 2012; Straube et al. 2011), and other semantically associated linguistic (Enrici, Adenzato, Cappa, Bara, \& Tettamanti, 2011; Graves, Desai, Humphries, Seidenberg, \& Binder, 2010; Mechelli, Josephs, Ralph, McClelland, \& Price, 2007) and non-linguistic (Saygin, Dick, Wilson, Dronkers, \& Bates, 2003) audio-visual stimuli. Highlevel factors modulate the response of this region to meaningful information including ambiguity (Rodd et al., 2005), familiarity (Straube, Green, Bromberger, \& Kircher, 2012), imagability (Sabsevitz, Medler, Seidenberg, \& Binder, 2005) and cross-modal congruency (Green et al. 2009; Rissman et al., 2003). Moreover, damage to this region has been associated with deficits in lexical-semantic processing in both modalities (Dronkers et al., 2004; Hart \& Gordon, 1990). The question is, what is the nature of representations and computations carried out within this region? 
As noted, recent functional imaging work, which showed no effect of sensory modality during the processing of auditory versus visual information, led to the view that the region represented high-level meaningful information in an amodal format. Other work hinted at representations that were more closely aligned with modalities such as the observation that responses to co-presented speech and gestures are stronger than responses to unimodal stimuli (Kircher et al. 2009; Willems \& Hagoort, 2007; Willems, Ozyurek, \& Hagoort, 2009). The present finding provides further evidence in support of the hypothesis that pMTG region codes information in a format that preserves, to some extent, the modal source of the inputs.

Of course, both the amodal and modality-linked hypotheses could be correct. Inputs to the pMTG from different sensory modalities could synapse on non-identical units, giving rise to the voxel-level modality preferences that we were able to detect in the present study. In addition, computations within this region could support the integration of information across modalities, with the result being sub-networks that code meaningful information in an amodal fashion. This would be similar to the intermixing of visual, motor, and visuomotor units in the macaque posterior parietal cortex (Sakata, Taira, Murata, \& Mine, 1995). Broadly speaking, our findings are consistent with Binder and Desai's classification of the pMTG region as "heteromodal cortex" (Binder \& Desai, 2011). 


\section{References}

Binder, J. R., Desai, R. H., Graves, W. W., \& Conant, L. L. (2009). Where is the semantic system? A critical review and meta-analysis of 120 functional neuroimaging studies. Cerebral Cortex, 19, 2767-2796.

Binder, J. R., \& Desai, R. H. (2011). The neurobiology of semantic memory. Trends in Cognitive Science, 15, 527-536.

Binder, J. R., Frost, J. A., Hammeke, T. A., Cox, R. W., Rao, S. M., \& Prieto, T. (1997). Human brain language areas identified by functional magnetic resonance imaging. The Journal of Neuroscience, 17, 353-362.

Binder, J. R., Swanson, S. J., Hammeke, T. A., \& Sabsevitz, D. S. (2008). A comparison of five fMRI protocols for mapping speech comprehension systems. Epilepsia, 49, 1980-1997.

Chang, C.-C., \& Lin, C.-J. (2011). LIBSVM : a library for support vector machines. $A C M$ Transactions on Intelligent Systems and Technology, 2:27.

Chen, G., Saad, Z.S., Nath, A.R., Beauchamp, M.S., Cox, R.W. (2012). FMRI group analysis combining effect estimates and their variances. Neuroimage, 60, 747-765.

. Computers in Biology and Medicine, 41, 1142-1155.

Chertkow, H., Bub, D., Deaudon, C., \& Whitehead, V. (1997). On the status of object concepts in aphasia. Brain and Language, 58, 203-232.

Courtin, C., Jobard, G., Vigneau, M., Beaucousin, V., Razafimandimby, A., Herve, P.-Y., Mellet, E., Zago, L. Petit, L., Mazoyer, B., \& Tzourio-Mazoyer, N. (2011). A common neural system is activated in hearing non-signers to process French sign language and spoken French. Brain Research Bulletin, 84, 75-87. 
Cox, R. W. (1996). AFNI: software for analysis and visualization of functional magnetic resonance neuroimages. Computers and Biomedical Research, 29, 162-173.

Damasio, A. R. (1989a). The brain binds entities and events by multiregional activation from convergence zones. Neural Computation, 1, 123-132.

Damasio, A. R. (1989b). Time-locked multiregional retroactivation: a systems-level proposal for the neural substrates of recall and recognition. Cognition, 33, 25-62.

Dick, A. S., Mok, E. H., Beharelle, A. R., Goldin-Meadow, S., \& Small, S. L. (2014). Frontal and temporal contributions to understanding the iconic co-speech gestures that accompany speech. Human Brain Mapping, 35, 900-917.

Dronkers, N. F., Wilkins, D. P., Valin, R. D. V., Redfern, B. B., \& Jaeger, J. J. (2004). Lesion analysis of the brain areas involved in language comprehension. Cognition, 92, 145-177.

Emmorey, K., Grabowski, T., McCullough, S., Damasio, H., Ponto, L., Hichwa, R., \& Bellugi, U. (2004). Motor-iconicity of sign language does not alter the neural systems underlying tool and action naming. Brain and Language, 89, 27-37.

Enrici, I., Adenzato, M., Cappa, S., Bara, B. G., \& Tettamanti, M. (2011). Intention processing in communication: a common brain network for language and gestures. Journal of Cognitive Neuroscience, 23, 2415-2431.

Forman, S. D., Cohen, J. D., Fitzgerald, M., Eddy, W. F., Mintun, M. A., \& Noll, D. C. (1995). Improved assessment of significant activation in functional magnetic resonance imaging (fMRI): use of a cluster-size threshold. Magnetic Resonance in Medicine, 33, 636-647.

Friston, K. J., Penny, W. D., \& Glaser, D. E. (2005). Conjunction revisited. NeuroImage 25, 661667. 
Graves, W. W., Desai, R., Humphries, C., Seidenberg, M. S., \& Binder, J. R. (2010). Neural systems for reading aloud: a multiparametric approach. Cerebral Cortex, 20, 1799-1815.

Green, A., Straube, B., Weis, S., Jansen, A., Willmes, K., Konrad, K., \& Kircher, T. (2009). Neural integration of iconic and unrelated coverbal gestures: a functional MRI study. Human Brain Mapping, 30, 3309-3324.

Hart, J., \& Gordon, B. (1990). Delineation of single-word semantic comprehension deficits in aphasia, with anatomical correlation. Annals of Neurology, 27, 226-231.

Haxby, J. V., Gobbini, M. I., Furey, M. L., Ishai, A., Schouten, J. L., \& Pietrini, P. (2001). Distributed and overlapping representations of faces and objects in ventral temporal cortex. Science, 293, 2425-2430.

Hickok, G., \& Poeppel, D. (2007). The cortical organization of speech processing. Nature Reviews Neuroscience, 8, 393-402.

Hillis A.E., \& Caramazza A. (1991). Category-specific naming and comprehension impairment: a double dissociation. Brain, 114, 2081-2094.

Humphries, C., Binder, J. R., Medler, D. A., \& Liebenthal, E. (2007). Time course of semantic processes during sentence comprehension: an fMRI study. NeuroImage, 36, 924-932.

Kertesz A., Lau W.K., \& Polk M. (1993) The structural determinants of recovery in Wernicke's aphasia. Brain and Language, 44, 153-164.

Kircher, T., Straube, B., Leube, D., Weis, S., Sachs, O., Willmes, K., Konrad, K., \& Green, A. (2009). Neural interaction of speech and gesture: differential activations of metaphoric coverbal gestures. Neuropsychologia, 47, 169-179. 
Kriegeskorte, N. R., Goebel, R., \& Bandettini, P. (2006). Information-based functional brain mapping. Proceedings of the National Academy of Sciences of the United States of America, $103,3863-3868$.

Lunneborg, C. E. (2000). Data Analysis by Resampling: Concepts and Applications. Pacific Grove, CA: Duxbury Press.

Mechelli, A., Josephs, O., Ralph, M. A. L., McClelland, J. L., \& Price, C. J. (2007). Dissociating stimulus-driven semantic and phonological effect during reading and naming. Human Brain Mapping, 28, 205-217.

Miglioretti, D. L., \& Boatman, D. (2003). Modeling variability in cortical representations of human complex sound perception. Experimental Brain Research, 153, 382-387.

Norman, K. A., Polyn, S. M., Detre, G. J., \& Haxby, J. V. (2006). Beyond mind-reading: multivoxel pattern analysis of fMRI data. Trends in Cognitive Science, 10, 424-430.

Okada, K., Rong, F., Venezia, J., Matchin, W., Hsieh, I., Saberi, K., Serences, J., \& Hickok, G. (2010). Hierarchical organization of human auditory cortex: evidence from acoustic invariance in the response to intelligible speech. Cerebral Cortex, 20, 2486-2495.

Rissman, J., Eliassen, J.C., \& Blumstein, S.E. (2003). An event-related FMRI investigation of implicit semantic priming. Journal of Cognitive Neuroscience, 15, 1160-1175.

Rodd, J. M., Davis, M. H., \& Johnsrude, I. S. (2005). The neural mechanisms of speech comprehension: fMRI studies of semantic ambiguity. Cerebral Cortex, 15, 1261-1269.

Rogers, T. T., \& McClelland, J. L. (2004). Semantic Cognition: A Parallel Distributed Processing Approach. Cambridge, MA: MIT Press.

Sabsevitz, D. S., Medler, D. A., Seidenberg, M., \& Binder, J. R. (2005). Modulation of the semantic system by word imageability. NeuroImage, 27, 188-200. 
Sakata, H., Taira, M., Murata, A., \& Mine, S. (1995). Neural mechanisms of visual guidance of hand action in the parietal cortex of the monkey. Cerebral Cortex, 5, 429-438.

Saygin, A. P., Dick, F., Wilson, S., Dronkers, N. F., \& Bates, E. (2003). Neural resources for processing language and environmental sounds: evidence from aphasia. Brain, 126, 928-945.

Straube, B., Green, A., Bromberger, B., \& Kircher, T. (2011). The differentiation of iconic and metaphoric gestures: common and unique integration processes. Human Brain Mapping, 32, $520-533$.

Straube, B., Green, A., Weis, S., \& Kircher, T. (2012). A supramodal neural network for speech and gesture semantics: an fMRI study. PLoS One, 7, e51207. doi:10.1371/journal.pone.0051207.

Vapnik, V. N. (1995). The Nature of Statistical Learning Theory. New York: Springer-Verlag.

Willems, R. M., \& Hagoort, P. (2007). Neural evidence for the interplay between language, gesture, and action: a review. Brain and Language, 101, 278-289.

Willems, R. M., Ozyurek, A., \& Hagoort, P. (2009). Differential roles for left inferior frontal and superior temporal cortex in multimodal integration of action and language. NeuroImage, 47, 1992-2004.

Xu, J., Gannon, P. J., Emmorey, K., Smith, J. F., \& Braun, A. R. (2009). Symbolic gestures and spoken language are processed by a common neural system. Proceedings of the National Academy of Sciences of the United States of America, 106, 20664-20669. 
REVIEWS and responses (indented)

Reviewer: 2

Comments to the Author

In this manuscript the authors reanalyze fMRI data from a previous study (Xu et al.). In the original paper, $\mathrm{Xu}$ and colleagues used univariate analyses to investigate neural responses to meaningul gestures and spoken versions of the same gestures; no differences were observed in posterior temporal cortex, leading the authors of the original study to suggest posterior temporal cortex was involved in amodal concept representation. In the current analyses of the data, MVPA shows that regions of posterior temporal cortex can distinguish between presentations in visual and auditory conditions, suggesting that - contrary to the conclusions of he first paper - it represents information from both modalities.

The issue of modality specific vs. amodal representations of concepts is a critical one, and I applaud the authors' reanalysis of an existing dataset that can speak to the issue. MVPA is appropriate here and can nicely add to our knowledge. That being said, I had some questions and comments on both the theory and approach of the curent manuscript that dampened my enthusiasm.

\section{MAJOR COMMENTS}

-It would be helpful if the authors were more specific regarding the anaotmical localization of their findings. They refer to the "left posterior temporal region" or "portions of the left posterior temporal lobe", However, there are ways to characterize this more precisely based on presumed cytoarthitecture, resting state parcellations, or simply other functional studies available to the authors that can serve as "localizers". I appreciate that there may still be some ambiguity, but a more accurate description would help us as readers get a better handle on the parameters of this variability (i.e., is it STS? MTG? MT?). In the discussion the authors start to use pMTG, but it is unclear to me whether this is intended to convey a different level of specificy than in the abstract and introduction.

-On a related note, nothing is mentioned regarding the relationship of the current findings to those of Beauchamp (e.g. 2004, etc.) and others who have characterized posterior STS (bordering pMTG) as reflecting inputs from multiple modalities. This includes some of the current authors in the context of audiovisual speech (Okada et al., 2013). These omissions are important because the current results are presented as shifting how the field views "posterior temporal regions" from amodal to something more heteromodal. However, I think it is farier to say that many people already assume at least some posterior temporal regions reflect inputs form multiple modalities (especially auditory and visual). So, the new analysis is informative and clears up the previous results, but I think it fits well within a rather large body of existing relevant literature. This perspective (and a large number of relevant pulications) is absent in the current version of the manuscript.

-Similarly, the relationship of the current findings to the perspective of Binder and colleagues is somewhat unclear. Although Binder et al. focus on the angular gyrus, my reading of their work is that angular gyrus is well-suited for heteromodal function in part because of its patterns of anatomical connectivity and evolutionary expansion, characteristics that are shared with regions of temporal cortex (i.e. MTG). I think they would characterize both angular gyrus and MTG as "heteromodal". In the introduction on p. 4, the authors seem to invoke Binder's 2009 meta-analysis as supporting an "amodal" account, which they contrast with "heteromodal". By the end of the discussion the authors 
indeed state that their results are consistent with Binder and others on MTG being heteromodal. However, this still leaves the motivations for the study as explained in the introduction muddy at best. Perhaps if the authors could do a more thorough job making the case for an amodal region in posterior MTG/STS that marshalls more evidence than Xu et al. it would more forcefully emphasize the need for clarification.

-The suggestion of potential gradients for modality preference is certainly interesting, but also qualitative (I didn't see any statistics on this) and thus speculative. I agree it could be included, but the langauge should make it clear that no firm conclusions can be drawn (unless the authors are able to statistically assess gradient-ness, which would be great).

-All told, I found the theoretical framing of the experiment to be lacking - in the end I wasn't sure whow theory was advanced compared to many of the studies cited in the discussion by the authors, and others which seem to have been ommitted (with the exception of Xu et al., the inferences of which the current work clearly argues against).

\section{MINOR COMMENTS}

-p. 5 The authors refer to their "more conservative" methods - it wasn't clear to me what was more conservative. Maybe they mean to say "different"?

-p. 6 Please note age, sex, and hearing of the participants.

-p. 6 What version of AFNI was used?

-p. 6 How were the brains spatially normalized?

-p. 7 What smoothness value was used for AlphaSim for each contrast?

-p. 7 "Only clusters showing statistical significance in both contrasts were included..." - I assume this means that the overlap of clusters from significant voxels were used as the ROI, which is fine, but is different than somehow combining the clusters in their entirety. Can you clarify the wording here?

-p. 15-16 - I don't think it odd that the univariate and multivariate results are different. The authors may want to discuss this, but I would at least move this point to later in the discussion, and lead with items of more theoretical importance.

-A minor point, but in general I think it is clearer to use "univariate" instead of GLM for the univariate analyses. (One could in principle do multivariate analyses on parameter estimates obtained from a GLM, for example.)

\section{References:}

Beauchamp MS, Argall BD, Bodurka J, Duyn JH, Martin A (2004) Unraveling multisensory integration: patchy organization within human STS multisensory cortex. Nat Neurosci 7:1190-1192.

Okada K, Venezia JH, Matchin W, Saberi K, Hickok G (2013) An fMRI study of audiovisual speech perception reveals multisensory interactions in auditory cortex. PLoS One 8:e68959.

Reviewer: 3 
Comments to the Author

I think this article presents a potentially important correction to the literature and should be published somewhere. I particularly like that some of the original authors are involved in the reanalysis and reinterpretation of their conclusions.

One thing I don't like as much about the paper is the kind of tacit assumption that the MVPA results are telling us something fundamental about the microstructure of representations in this brain region. It could just be the case that there are small differences between these stimuli in terms of their overall activation in this region and that is leading to differentiation of the two categories.

Response: Our methods subtract out overall activation levels.

Also, it could just be that some subjects activate more for one versus the other.

Response: MVPA is done on an individual subject basis. This cannot explain the effects.

MVPA largely removes directional information, so all that is required is for conditions to be distinguishable within each subject. However, given how previous efforts have focused on how this brain region activates the same for both types of information, I think showing that it distinguishes between them might be enough for a paper without having to make conclusions about microstructure or that this analysis is telling us anything other than that these types of information are distinguishable in this region.

Response: Our only real claim was that the region distinguishes activation in the region. The microstructure was a secondary observation.

Along these lines, one thing I kept wondering was whether there is any behavioral data from this study. This version doesn't have any presented and the original publication at PNAS doesn't seem to either. The reason why it might be important is if there are behavioral differences between the conditions in terms of how long people attend to each gloss (i.e., time-on-task). This could be critical for the interpretation of the results as MVPA will be sensitive to small differences in the HRF due to time on task such that a region that is actually amodal in terms of underlying representation may look like it distinguishes between conditions because of HRF duration differences.

Response: Incorrect. Activation amplitude is normalized so that global variation cannot drive classification. Further, the standard analysis showed that there is no amplitude difference overall.

The spatial organization of the classifier weights is intriguing and suggests perhaps a deeper characterization of the microstructure of this region than is implied by the classification analysis alone. However, I'm wondering whether there isn't some formal test that would be required to conclude that the spatial distribution of two signals is not equivalent? For example, how likely is the present distribution of classifier weights if one assumed the null hypothesis is that they are identically distributed? Without showing whether this organization could arise due to chance, it's tough to evaluate whether it means anything.

Response: It was just an observation that we thought would be interesting to show.

Another concern I have is about the ROI definition. Intuitively, taking the voxels that are jointly active for both conditions relative to controls $(G P+G E-G C+S P+S E-S C)>0$ SHOULD be orthogonal to the 
contrast comparing the two conditions to each other $[(G P+G E)-(S P+S E)]>0$. The problem is that most of the time it isn't because conditions differ in terms of their variance. For example, GP+GE might be more variable than SP+SE and produce both higher and lower activations than SP+SE. When this happens, the otherwise orthogonal contrast will tend to contain more activated voxels that are specific for the variable condition. This isn't a major problem if the data used to define the ROI are separate from those used to test the classifier. However, here it looks like the same data are used meaning that, if there are variance differences, you could expect differentiation between the conditions based on the ROI selection itself (without there being any true mean differences).

Response: Reasonable thoughts broadly, but in our particular case the ROI was defined precisely for NOT distinguishing between the activation conditions. Or put differently, the goal of the re-analysis was to see if there was any internal sensitivity to the different modalities *in the ROI that was defined by the original contrast* ${ }^{*}$ If one wants to argue that we found differences because there are differences, then that pretty much assumes the point we were trying to make. And again, we normalize overall activation levels across conditions.

When evaluating the strengths and weaknesses, the real question arises about what to do with all of the studies where similar conclusions have been reached based on equivalent activation in a region. I think nowadays one would be hard pressed to publish the original results because the field has greater appreciation for the fact that statistically indistinguishable activation does not mean that the brain region treats two conditions as identical. However, activation equivalence was clearly thought to be really important evidence just a few years ago and some "textbook" findings about language, object-recognition, and representation in general still hinge on results that really just showed that a brain region activated for two things. It's important to correct the literature, but it's not clear that every single study that made similar claims based on conjunction analyses or other tests of equivalent activation should merit a published reanalysis. Perhaps the authors could reframe this slightly as a general warning about such types of conclusions and the type of evidence they believe would be necessary to make them. That is, assuming they are like me and now agree that equivalent activation is meaningless in and of itself.

Response: So this is key: if no one believes that no difference means no difference anymore than the finding is indeed trivial and not appropriate for PBR. However, one could argue that our study evaluated precisely what "no difference" means in this case. 